\title{
Coexistence of superconductivity and spin-splitting fields in superconductor/ferromagnetic insulator bilayers of arbitrary thickness
}

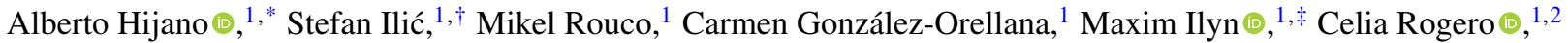 \\ P. Virtanen, ${ }^{3}$ T. T. Heikkilä $\odot,{ }^{3}$ S. Khorshidian $\left(\bullet,{ }^{4,5}\right.$ M. Spies $\odot,{ }^{5}$ N. Ligato, ${ }^{5}$ F. Giazotto,${ }^{5}$ \\ E. Strambini, ${ }^{5, \S}$ and F. Sebastián Bergeret ${ }^{1,2,6, \|}$ \\ ${ }^{1}$ Centro de Física de Materiales (CFM-MPC) Centro Mixto CSIC-UPV/EHU, E-20018 Donostia-San Sebastián, Spain \\ ${ }^{2}$ Donostia International Physics Center (DIPC), 20018 Donostia-San Sebastián, Spain \\ ${ }^{3}$ Department of Physics and Nanoscience Center, University of Jyväskylä, P.O. Box 35 (YFL), FI-40014 University of Jyväskylä, Finland \\ ${ }^{4}$ Department of Physics, College of Sciences, Yasouj University, Yasouj, 75914-353, Iran \\ ${ }^{5}$ NEST, Istituto Nanoscienze-CNR and Scuola Normale Superiore, I-56127 Pisa, Italy \\ ${ }^{6}$ Institute of Solid State Theory, University of Münster, D-48149 Münster, Germany
}

(Received 6 January 2021; revised 16 March 2021; accepted 28 April 2021; published 19 May 2021)

\begin{abstract}
Ferromagnetic insulators (FI) can induce a strong exchange field in an adjacent superconductor (S) via the magnetic proximity effect. This manifests as spin splitting of the BCS density of states of the superconductor, an important ingredient for numerous superconducting spintronics applications and the realization of Majorana fermions. A crucial parameter that determines the magnitude of the induced spin splitting in FI/S bilayers is the thickness of the $\mathrm{S}$ layer $d$ : In very thin samples, the superconductivity is suppressed by the strong magnetism. By contrast, in very thick samples, the spin splitting is absent at distances away from the interface. In this work, we calculate the density of states and critical exchange field of FI/S bilayers of arbitrary thickness. From here, we determine the range of parameters of interest for applications, where the exchange field and superconductivity coexist. We show that for $d>3.0 \xi_{s}$, the paramagnetic phase transition is always of the second order, in contrast to the first-order transition in thinner samples at low temperatures. Here $\xi_{s}$ is the superconducting coherence length. Finally, we compare our theory with the tunneling spectroscopy measurements in several $\mathrm{EuS} / \mathrm{Al} / \mathrm{AlO}_{x} / \mathrm{Al}$ samples. If the $\mathrm{Al}$ film in contact with the $\mathrm{EuS}$ is thinner than a certain critical value, we do not observe superconductivity, whereas, in thicker samples, we find evidence of a first-order phase transition induced by an external field. The complete transition is preceded by a regime in which normal and superconducting regions coexist. We attribute this mixed phase to inhomogeneities of the $\mathrm{Al}$ film thickness and the presence of superparamagnetic grains at the EuS/Al interface with different switching fields. The steplike evolution of the tunnel-barrier magnetoresistance supports this assumption. Our results demonstrate on the one hand, the important role of the S layer thickness, which is particularly relevant for the fabrication of high-quality samples suitable for applications. On the other hand, the agreement between theory and experiment demonstrates the accuracy of our theory, which, originally developed for homogeneous situations, is generalized to highly inhomogeneous systems.
\end{abstract}

DOI: 10.1103/PhysRevResearch.3.023131

\section{INTRODUCTION}

It was shown a long time ago [1], and confirmed in several later experiments [2-10], that a thin superconducting film (S), adjacent to a ferromagnetic insulator (FI), may exhibit

\footnotetext{
*ahijano001@ikasle.ehu.eus

†stefan.ilic@csic.es

${ }^{\ddagger}$ maxim.ilin@ehu.eus

§elia.strambini@sns.it

"fs.bergeret@csic.es
}

Published by the American Physical Society under the terms of the Creative Commons Attribution 4.0 International license. Further distribution of this work must maintain attribution to the author(s) and the published article's title, journal citation, and DOI. a spin-split density of states even at zero field. The splitting is due to the interfacial exchange interaction between the localized magnetic moments and the Al film's conduction band electrons. Even though back in the late 1980s and 1990s, this effect had attracted attention mainly from a fundamental research perspective [11], only recently superconductors with a spin-split density of states (DoS) are proposed for diverse applications, such as topological qubits using Majorana wires $[12,13]$, spin valves $[9,14]$, thermometry $[15,16]$, magnetometers $[17,18]$, caloritronic devices [19-21], thermoelectricity [22,23], and radiation detectors [24].

An ideal material combination for observing spin-split superconductivity at zero field is EuS/Al. This has been confirmed in numerous spectral measurements on EuS/Al samples, mainly grown by Moodera's group at MIT [2,4,8,9]. It is understood that the splitting size at the interface is proportional to the interfacial exchange field, which in turn is 
proportional to the averaged magnetic moment of the EuS $[8,25]$. Thus, one needs high-quality $\mathrm{S} / \mathrm{FI}$ interfaces for a sizable exchange field, avoiding a nonmagnetic interlayer between the two materials. It is also known that the effective splitting field decays away from the interface over the superconducting coherence length [26]. Thus, for applications that require an almost homogeneous splitting, the $\mathrm{S}$ layers have to be thin enough. On the other hand, the induced exchange cannot be too strong because it would destroy the superconductivity [27,28]. The difficulty then lies in manufacturing superconducting films thin enough to have a sufficiently large splitting field, but at the same time, the field has to be weak enough in order not to suppress the superconducting state.

Indeed, FI/S systems with a $\mathrm{S}$ layer thinner than the superconducting coherence length behave as homogeneous superconductors in a Zeeman field. In this case, the wellestablished theory of a paramagnetic phase transition to the normal state applies $[29,30]$. However, if the S layer's thickness is comparable to the superconducting coherence length, the spin-splitting field is nonhomogeneous, and hence the theory needs to be revised. The new theory has to connect the thin layer limit, in which the phase transition takes place, and the thick S layer limit, in which one expects no transition to the normal state for any value of the interfacial exchange field. Clearly, in this latter case, the splitting is negligible at the boundary opposite to the FI/S interface, and hence such a system is less suitable for applications requiring spin splittings. It is crucial for experiments to find the optimal values of the interfacial exchange field and the S-layer's thickness to simultaneously observe a well-defined superconducting gapped state and a sharp spin splitting of the quasiparticle peaks at zero field. Even though several works have studied the effect of a homogeneous spin-splitting field on the superconducting properties of the S layer in FI/S structures, there is no study, to the best of our knowledge, on the role of the $\mathrm{S}$ thickness on the spectral properties of FI/S junctions. ${ }^{1}$

This work addresses this problem and presents an exhaustive theoretical analysis of the spectral properties and phase transition of diffusive superconducting films of arbitrary thickness adjacent to a FI layer. The combination of the DoS and the phase diagram gives a complete picture of the system that can help to identify the range of parameters where superconductivity and a well-defined spin splitting coexist, which is the desired situation for applications. Moreover, we infer the nature of the phase transition at different temperatures from the nonmonotonic behavior of the critical exchange field and find a temperature-dependent critical thickness above which there is no phase transition, regardless of the value of the exchange field. Our work also includes the fabrication and transport measurements of $\mathrm{EuS} / \mathrm{Al} / \mathrm{AlO}_{\mathrm{x}} / \mathrm{Al}$ junctions. The

\footnotetext{
${ }^{1}$ In Refs. [59,60] a possible phase transition to the FFLO state has been studied in all-metallic ferromagnet superconductor and superconductor-ferromagnet normal metal structures with different thickness. According to these works, the FFLO may appear when the conductivity of the nonsuperconducting region is much larger than the conductivity of $\mathrm{S}$ in the normal state. In our case we consider a ferromagnetic insulator and hence we are in the opposite limit. Therefore, we may ignore the FFLO state [42,58].
}

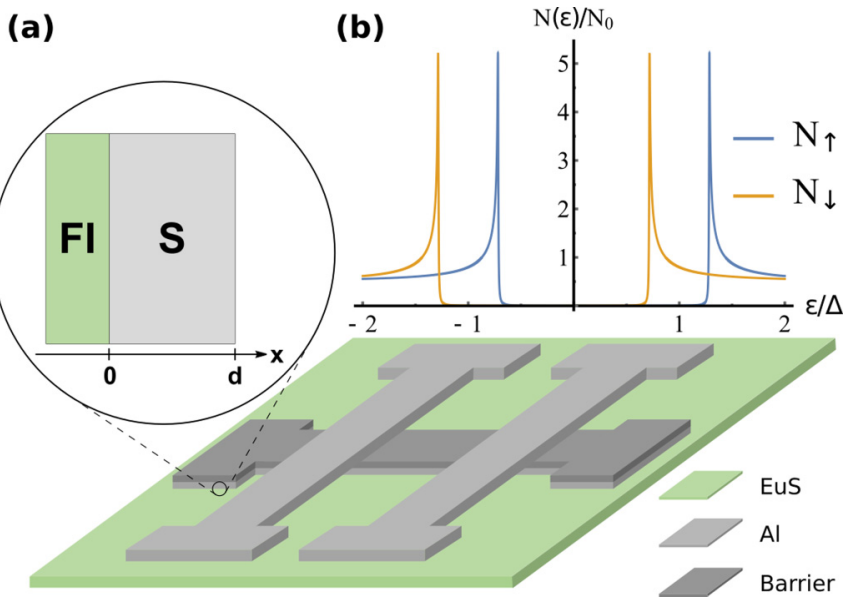

FIG. 1. (a) Experimental setup and schematic view of the FI/S bilayer. The $\mathrm{S}$ layer in contact with the FI has a thickness $d$. See Sec. V for additional details on the experiment. (b) DoS of a homogeneous spin-split superconductor.

Al film next to the EuS layer exhibits a spin-split DoS. By applying an external magnetic field the spin-splitting changes. At $30 \mathrm{mK}$ we observe a clear signature of a first-order phase transition when the splitting field equals to $\Delta_{0}^{*} / \sqrt{2}$, where $\Delta_{0}^{*}$ is the superconducting gap at zero exchange field in the presence of magnetic impurities. We also show the magnetic field dependence of the resistance at very low temperatures, which suggests the presence of a mixed phase with superconducting and normal parts at certain field ranges. This mixed phase is confirmed by the good agreement between the measured differential conductance and the results of our theoretical model.

This paper is organized as follows: In Sec. II we present the basic equations describing diffusive superconductors and the boundary conditions at the FI/S interface. In Sec. III we obtain the density of states (DoS) for different values of the thickness of the S layer. In Sec. IV we calculate the critical exchange field of the system and discuss the nature of the paramagnetic phase transition. In Sec. V, we present details of the fabrication of $\mathrm{EuS} / \mathrm{Al} / \mathrm{AlO}_{\mathrm{x}} / \mathrm{Al}$ junctions and the tunneling spectroscopy measurements, which we compare to our theoretical results. We summarize the results in Sec. VI.

\section{MODEL AND FORMALISM}

In this section, we introduce the basic equations determining the spectral properties of a FI/S bilayer [see the inset of Fig. 1(a)] for arbitrary values of the exchange field and $\mathrm{S}$ layer thickness $d$. We assume that the system is homogeneous in the directions parallel to the interface.

We describe the system using the Green's function (GF) technique. In the case under consideration, the spatial scales over which the superconducting properties vary are much larger than the Fermi wavelength. Moreover, all relevant energies involved are smaller than the Fermi energy. In this case, one can use the quasiclassical approximation, which simplifies the problem considerably in hybrid systems [31-36]. Because we are dealing with superconductivity and spindependent fields, the quasiclassical Green's function $\breve{g}$ is a $4 \times 4$ matrix in Nambu-spin space. The density of states is 
related to the retarded GF $\breve{g}^{R}$ as

$$
N(\boldsymbol{r}, \varepsilon)=\frac{N_{0}}{4} \operatorname{Re} \operatorname{Tr}\left\{\tau_{3} \breve{g}^{R}(\boldsymbol{r}, \varepsilon)\right\},
$$

where $N_{0}$ is the density of states at the Fermi level in the normal state and $\operatorname{Tr}$ denotes the trace over Nambu and spin spaces.

The GF in Eq. (1) has to be determined from the quasiclassical equations, which in the dirty limit reduce to a diffusivelike equation, known as the Usadel equation [33] $]^{2}$

$$
D \partial_{x}\left(\check{g} \partial_{x} \check{g}\right)+\left[i \varepsilon \tau_{3}+\Delta \tau_{2}-i \boldsymbol{h} \cdot \boldsymbol{\sigma} \tau_{3}-\check{\Sigma}, \check{g}\right]=0,
$$

and the normalization condition, $\breve{g}^{2}=\breve{1}$. Here, $D$ is the diffusion constant, $\varepsilon$ is the energy, $\Delta$ is the superconducting order parameter, and $\boldsymbol{h}$ is the exchange field. The latter is only finite at the FI/S interface, and we approximate it as $\boldsymbol{h}=h a \delta(x) \hat{\boldsymbol{z}}$, where $h$ is the exchange field, and $a$ is the thickness of an effective layer over which the exchange interaction is finite [25]. The matrices $\sigma_{i}$ and $\tau_{i}(i=1,2,3)$ in Eq. (2) are the Pauli matrices in spin and Nambu space, respectively. $\check{\Sigma}$ is the selfenergy term, which describes different scattering processes, such as magnetic and spin-orbit impurities [11,37,38]. Most of the experiments on spin-split superconductors, including those in the present work, are made using Al layers, for which the spin-orbit coupling can be neglected. Therefore we only consider the spin-flip relaxation processes, described by the self-energy term:

$$
\check{\Sigma}_{\text {sf }}=\frac{\sigma_{i} \tau_{3} \check{g}_{3} \sigma_{3}}{8 \tau_{\mathrm{sf}}} a \delta(x)
$$

where $\tau_{\mathrm{sf}}$ is the spin-flip relaxation time, and sum over repeated indices is implied. Even though the $\mathrm{Al}$ films often have a tiny concentration of homogeneously distributed magnetic impurities, the main source of magnetic disorder and a sizable spin-flip relaxation is the FI/S interface [11]. This assumption is supported by contrasting our model with the experimental data presented in Sec. V. As with the exchange field, we then assume that the self-energy, Eq. (3), is only finite at the interface. Thus, the Usadel equation (2) in the superconducting layer does not contain spin-dependent terms and becomes

$$
D \partial_{x}\left(\check{g} \partial_{x} \check{g}\right)+\left[i \varepsilon \tau_{3}+\Delta \tau_{2}, \check{g}\right]=0 .
$$

Both the exchange and spin-relaxation terms enter the boundary conditions at the FI/S interface $(x=0)$, which can be obtained by integrating Eq. (2) in a small region around the interface:

$$
\left.\check{g} \partial_{x} \check{g}\right|_{x=0}=\left.\frac{1}{D}\left[i h a \sigma_{3} \tau_{3}+\frac{\sigma_{i} \tau_{3} \check{g} \tau_{3} \sigma_{i}}{8 \tau_{\mathrm{sf}}} a, \breve{g}\right]\right|_{x=0} .
$$

The second term in the commutator stems from the spin-flip processes and mixes GF components with opposite spins.

The spectral current vanishes at the boundary with vacuum or an insulator. This implies the boundary condition

$$
\left.\check{g} \partial_{x} \check{g}\right|_{x=d}=0 .
$$

\footnotetext{
${ }^{2}$ Here and throughout the paper we set $\hbar=1$.
}

The quasiclassical GF is then determined from Eqs. (4)-(6) and the normalization condition. This set of equations is complemented by the self-consistency relation for $\Delta$. In Secs. III and IV, we solve this boundary problem numerically and compute the DoS and the critical temperature and critical exchange field for FI/S bilayers, for arbitrary values of the interfacial exchange field and $\mathrm{S}$ layer thickness.

\section{DENSITY OF STATES OF A FI/S BILAYER}

In this section, we study the spatial dependence of the DoS for different thicknesses of the superconducting layer. We solve the boundary problem (4)-(6) and evaluate the DoS using Eq. (1).

We start analyzing the thin-film limit. The characteristic length scale of the Usadel equation is the superconducting coherence length, which at low temperatures is approximately given by $\xi_{0}=\sqrt{D / \Delta}$. In the thin-layer limit the thickness of the superconductor $d$ is much smaller than $\xi_{0}$, so we may assume that $\breve{g}$ is constant. The effective value for the exchange field, $\bar{h}$ and the spin-flip rate, $1 / \bar{\tau}_{\mathrm{sf}}$ can then be obtained by integrating Eq. (4) over the $\mathrm{Al}$ thickness and the boundary conditions Eqs. (5)-(6)

$$
\begin{gathered}
\bar{h}=h a / d, \\
\bar{\tau}_{\mathrm{sf}}^{-1}=\tau_{\mathrm{sf}}^{-1} a / d .
\end{gathered}
$$

In the absence of spin-flip scattering, $\bar{\tau}_{\mathrm{sf}}^{-1}=0$, the spinresolved DoS for spin-up $(\uparrow)$ and spin-down $(\downarrow)$ quasiparticles has the form as for a homogeneous superconductor in a Zeeman field:

$$
N_{\uparrow, \downarrow}(\varepsilon, \bar{h})=\frac{N_{0}}{2} \operatorname{Re} \frac{|\varepsilon \mp \bar{h}|}{\sqrt{(\varepsilon \mp \bar{h})^{2}-\Delta^{2}}} .
$$

In Fig. 1(b) we show the corresponding DoS of a homogeneous spin-split superconductor given by this equation. The homogeneous exchange field $\bar{h}$ induces a spin splitting of the density of states, such that the states for each spin direction are raised or lowered in energy.

The DoS of superconductors of intermediate $\left(d=0.5 \xi_{0}\right)$ and large thicknesses $\left(d=3 \xi_{0}\right)$ are calculated numerically, and shown in Fig. 2. Unlike the homogeneous thin layer limit, the DoS in this case varies in space. For intermediate $S$ layers, the spin-splitting remains almost constant along the sample, but the spin splitting vanishes away from the FI/S interface in thick samples. This is as expected, since in the thick sample limit we should recover the BCS density of states without any spin splitting at distances far away from the interface. Another notable effect is that as we move away from the interface, the height of the inner peaks is reduced, while the outer peaks increase in size.

Comparing Figs. 2(a) and 2(b) we see that for a given value of the interfacial exchange field $h$, the spin splitting decreases by increasing thickness of the $\mathrm{S}$ layer. The value of the spin splitting in the very thin superconductor limit is given by $2 \bar{h}$, where $\bar{h}$ is the average exchange field defined in Eq. (7). Extracting the spin splitting from the panels, we show that the splitting at $x=0$ is well approximated by this expression even for thick samples. 

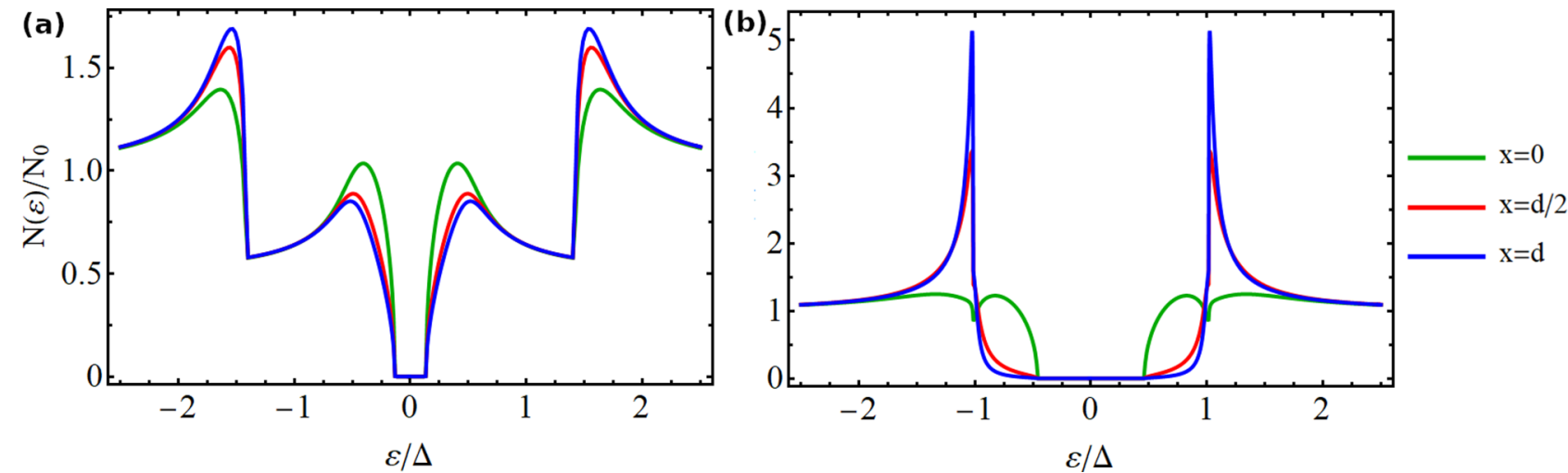

FIG. 2. DoS for (a) a superconductor of intermediate thickness $\left(d=0.5 \xi_{0}\right)$ and (b) thick superconductor $\left(d=3 \xi_{0}\right)$ at different distances from the FI/S interface: $x=0$ (green), $x=d / 2$ (red) and $x=d$ (blue). Note that the energy is normalized by the order parameter. The value of the exchange field is $h a / \xi_{0}=0.3 \Delta$, and we assume no magnetic impurities $\left(\tau_{\mathrm{sf}}^{-1}=0\right)$.

In Fig. 3(a) we show the DoS of a S layer of intermediate thickness for different values of the effective spin-flip rate [see Eq. (8)]. As the spin-flip rate is increased, the coherence peaks are smeared and the superconducting gap is suppressed. Above a threshold value of the spin-flip rate, the superconductor is driven to the normal state.

Increasing the value of the exchange field beyond some threshold value induces a phase transition from the superconducting state to the normal state. We analyze this transition in the next section.

\section{CRITICAL TEMPERATURE OF A FI/S BILAYER}

In this section we determine the critical temperature $T_{c}$ of the FI/S bilayers. In addition to the DoS, this is another experimentally accessible quantity, which is determined by measuring the resistance drop as a function of temperature.

Calculation of $T_{c}$ as a function of other system's parameters allows us to construct the phase diagram for a FI/S bilayer. For this sake we employ the Matsubara Green's functions, which are obtained from Eqs. (4)-(6) after the substitution $\varepsilon \rightarrow i \omega_{n}$. Here, $\omega_{n}=2 \pi T(n+1 / 2), n \in \mathbb{Z}$, are the Matsubara frequencies [39].
First, we assume a second-order phase transition and look for the solution of the Usadel equation at temperatures close to $T_{c}$. In this case one can linearize the problem by treating $\Delta$ perturbatively. Near $T_{c}$ the GF can be approximated by $\check{g}=$ $\operatorname{sgn}\left(\omega_{n}\right) \tau_{3}+i \hat{f} \tau_{2}$, where the anomalous function $\hat{f}$ satisfies the linearized Usadel equation:

$$
\xi_{s}^{2} \pi T_{c 0} \partial_{x x}^{2} \hat{f}-\left|\omega_{n}\right| \hat{f}+i \Delta=0,
$$

with $\xi_{s}^{2}=D /\left(2 \pi T_{c 0}\right)$, where $T_{c 0}$ is the critical temperature in the absence of the magnetic field and $\xi_{s}$ is the coherence length close to the critical temperature.

As discussed in Sec. II, the exchange field and spin-flip scattering rate enter the boundary condition at the $\mathrm{FI} / \mathrm{S}$ interface, which after linearization reads [see Eq. (5)]

$$
\begin{aligned}
& \left.\partial_{x} \hat{f}\right|_{x=0}=i \operatorname{sgn}\left(w_{n}\right) \kappa_{h} \sigma_{3} \hat{f}+\left.\kappa_{s f}\left(3 \hat{f}+\sigma_{i} \hat{f} \sigma_{i}\right)\right|_{x=0}, \\
& \left.\partial_{x} \hat{f}\right|_{x=d}=0,
\end{aligned}
$$

where $\kappa_{h}=a h /\left(\pi T_{c 0} \xi_{s}^{2}\right)$ and $\kappa_{\mathrm{sf}}=a /\left(8 \tau_{\mathrm{sf}} \pi T_{c 0} \xi_{s}^{2}\right)$. The order parameter $\Delta$ is then determined self-consistently from the gap equation [40]:

$$
\Delta \ln \frac{T_{c 0}}{T}=\pi T \sum_{\omega_{n}}\left(\frac{\Delta}{\left|\omega_{n}\right|}+\frac{i}{2} \operatorname{Tr} \hat{f}\right) .
$$
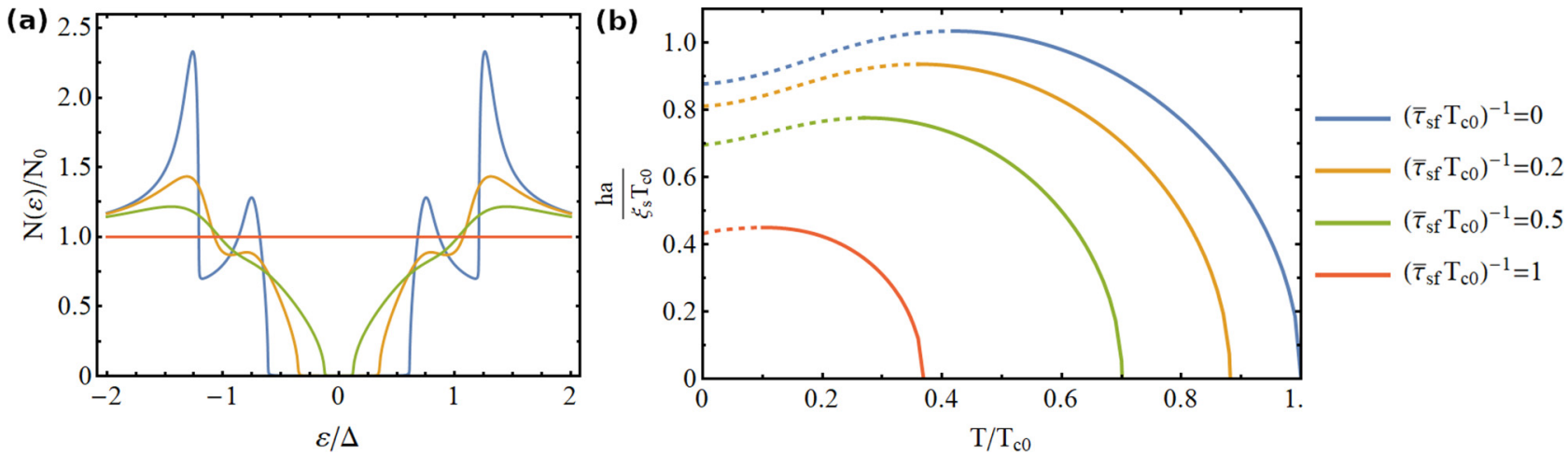

FIG. 3. (a) DoS at $x=d$ for different values of the spin-flip relaxation rate. The samples have a thickness $d=\xi_{s}$, and the value of the exchange field is $h a / \xi_{s}=0.5 T_{c 0}$. (b) Phase boundary for the second-order phase transition. The solid part of the lines represent the critical exchange field for the second-order transition, while the dashed lines correspond to the temperature range where the first-order phase transition occurs. 
(a)

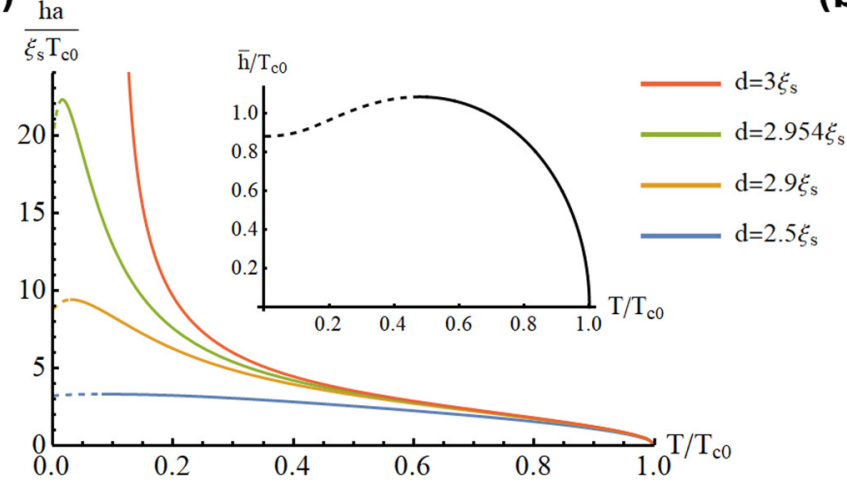

(b)

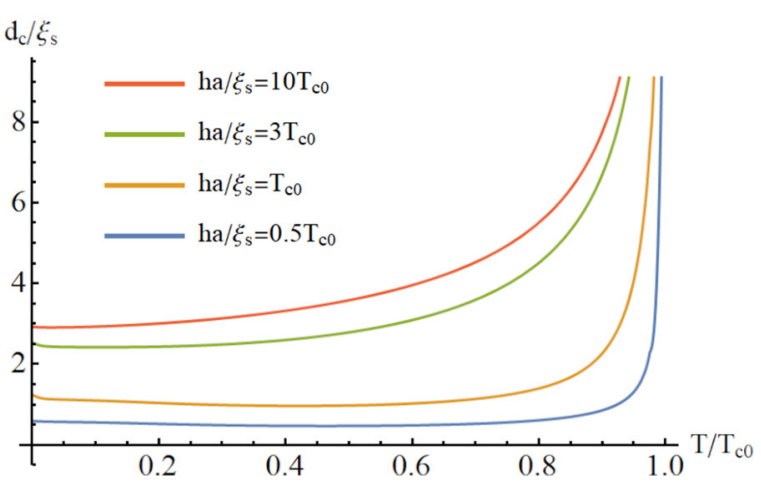

FIG. 4. (a) Critical exchange field for different thicknesses of the superconductor and (b) temperature dependence of the critical thickness for different exchange fields in the magnetic impurity-free limit $\left(\tau_{\mathrm{sf}}^{-1}=0\right)$. The inset in (a) corresponds to the critical exchange field in the thin limit (homogeneous case). The dashed part corresponds to the temperature region for which the transition is of first order.

From this equation we can determine the critical temperature and field. In order to solve this problem we use the ansatz for $\hat{f}$ prescribed by the so-called multimode method developed by Fominov et al. [41] (see Appendix A for details).

The nature of the paramagnetic phase transition of a FI/S bilayer depends on the temperature, but also on the degree of disorder of the metal. Namely, Tokuyasu et al. [26] have shown that in the clean limit, this phase transition is always of the second order. However, a recent work established that even a small amount of impurity scattering restores the possibility of a first-order phase transition [42]. We assume here the diffusive limit, which is well justified for $\mathrm{Al}$ films due to their intrinsic disorder.

In the thin layer limit, $d \ll \xi_{s}, \mathrm{FI} / \mathrm{S}$ bilayers are homogeneous, and evaluating the gap equation (12) yields the standard expression for the paramagnetically limited secondorder phase transition in the absence of magnetic impurities [40]

$$
\ln \frac{T_{c 0}}{T}=\operatorname{Re}\left[\psi\left(\frac{1}{2}+\frac{i \bar{h}}{2 \pi T}\right)-\psi\left(\frac{1}{2}\right)\right] .
$$

Here, $\psi$ is the digamma function. Importantly, Eq. (13) only holds when the temperature is higher than $T^{*}=0.56 T_{c 0}$. At lower temperatures, the phase transition is of the first order, which can be readily proved by analyzing the free energy of the superconductor [40]. At $T=0$, the critical field is given by the Chandrasekhar-Clogston limit $\bar{h}=\Delta_{0} / \sqrt{2}[27,28]$. The nature of the phase transition can, however, be inferred even without knowing the free energy, from the shape of the $\bar{h}(T)$ critical line. Namely, below $T^{*}$ the critical line exhibits nonmonotonic behavior, and the critical temperature is a double-valued function of the field [see Fig. 4(a)], but the smaller solution is physically unstable. Therefore, the onset of the first-order phase transition coincides with the nonmonotonic features in the $h(T)$ diagram. We assume that the multivaluedness of the solution surface $\Delta(h, T)$ is correlated with the nonmonotonicity of $h(T)$ to identify the nature of the phase transition in thick FI/S bilayers.

We first consider the effect of spin-flip relaxation. In Fig. 3 we show the DoS and $h(T)$ diagram of a $\mathrm{S}$ layer of intermediate thickness $\left(d=\xi_{s}\right) . \bar{\tau}_{\mathrm{sf}}$ is the effective value for the spin-flip rate, given by Eq. (8).
The critical exchange field is suppressed by magnetic impurities as shown in Fig. 3(b). If the spin-flip rate is strong enough, the superconductor is driven to the normal state. For example, for an exchange field value of $\bar{h}=0.5 T_{c 0}$ and a spin-flip scattering of $\left(\bar{\tau}_{\mathrm{sf}} T_{c 0}\right)^{-1}=1$ the system would be in the normal state. This is reflected in the DoS shown in Fig. 3(a).

In the following, we focus on the magnetic impurity-free limit, $\tau_{\mathrm{sf}}^{-1}=0$. In Fig. 4 we show the $h(T)$ diagrams for different values of the thicknesses of the superconducting layer. In order to study the effect of the thickness of the superconductor on the transition, we define a dimensionless exchange field as $h a /\left(\xi_{s} T_{c 0}\right)$, so that the normalizing factor is thickness independent. As shown in the left panel of Fig. 4: the thicker the sample, the higher the critical exchange field. The exchange field is located at the FI/S interface, so the influence of the interaction will diminish as we move away from the interface. Thus, the exchange field required to induce a phase transition increases monotonically with the thickness of the sample. Notably, there is a region where the critical temperature is a double-valued function of the field where, as explained above, the phase transition is of the first order for the lower temperatures. As the thickness of the sample is increased, the maximum critical exchange field is shifted towards lower temperatures, and the temperature range in which a first-order transition occurs is reduced accordingly.

For thicknesses larger than a certain value $d^{*}$, the critical exchange field diverges at low temperatures, which means that the sample is in the superconducting state at $T=0$ for all values of the interface exchange field. In other words, if the sample is thick enough, the exchange field cannot induce a phase transition to the normal state. Therefore, depending on the thickness of the $S$ layer, our numerical analysis suggests that the phase transition at zero temperature is either of the first order or does not take place.

The right panel of Fig. 4 shows the critical thickness at which the phase transition occurs for different values of the exchange field. The metal is in the superconducting state if its thickness lies above the phase boundary. In the absence of an exchange field the metal is in the superconducting state for temperatures lower than $T_{c 0}$ and in the normal state for higher temperatures, regardless of the thickness. 
The value of the critical thickness increases as the value of the exchange field is increased. As shown in Fig. 4(b), the $h$ dependence of the critical thickness becomes weaker for large exchange fields, so that the curves approach a limiting behavior for large fields. By analyzing the $T \rightarrow 0$ limit, we obtained that the maximum thickness for which the critical exchange field exists and a phase transition occurs is (see Appendix B for the derivation)

$$
d^{*}=\sqrt{\frac{\gamma_{E}}{2}} \pi \xi_{s} \approx 3.0 \xi_{s},
$$

where $\gamma_{E} \approx 1.781$ is the exponent of the Euler-Mascheroni constant. Note that the value $d^{*}$ of the critical thickness was already reported by Fominov et al. [41] for an all metallic ferromagnet/superconductor bilayer at $T \rightarrow 0$ for a certain combination of junction parameters. We have demonstrated that this is also valid for a FI/S junction. If the thickness of the $\mathrm{S}$ layer is further increased, the critical exchange field no longer diverges at $T=0$, but at a higher temperature. In the next section we present experimental results and contrast them with the previous theoretical analysis.

\section{EXPERIMENTS AND DISCUSSION}

In this section, we compare the experimental results of $\mathrm{Al} / \mathrm{EuS}$ bilayers with our model's predictions. To do that, we have fabricated four $\mathrm{Al} / \mathrm{AlO}_{\mathrm{x}} / \mathrm{Al} / \mathrm{EuS} /$ silica tunnel junctions (from top to bottom). The distribution of the layers in the fabricated tunnel junctions is shown in Fig. 1. A ferromagnetic insulator $(\mathrm{EuS})$ layer is grown on the polished fused silica substrate. The bottom Al wire (denoted as $\mathrm{S}$ in the picture) is in contact with the EuS layer, forming the FI/S interface. Two top $\mathrm{Al}$ wires are rotated $90^{\circ}$ with respect to the bottom, these wires form the superconducting tunneling probes used to perform the spectroscopy measurements. The barrier between the two $\mathrm{Al}$ layers is made of nonstoichiometric aluminum oxide $\left(\mathrm{AlO}_{\mathrm{x}}\right)$. A thickness of the layers was monitored via quartz microbalance that was initially calibrated by means of the $\mathrm{x}$-ray reflectivity measurements.

We fabricated four samples, with two junctions each, where we performed tunneling spectroscopy measurements at different temperatures with and without external magnetic fields in all samples. The tunneling spectroscopy of the junctions was done at temperatures down to $30 \mathrm{mK}$ in a filtered cryogen-free dilution refrigerator. The I-V curves were measured in a standard dc four-wire configuration [8], from which the differential conductance was calculated via numerical differentiation. From the value of the experimentally measured $T_{c}$ we can estimate the superconducting coherence length. Previous studies on thinner Al layers, $4 \mathrm{~nm}$, showed larger values of the critical temperature $\left(T_{c} \sim 2.3 \mathrm{~K}\right)^{3}$ [43]. In those cases the coherence length was estimated as $\xi_{s} \sim 35 \mathrm{~nm}$. Our films exhibit a smaller $T_{c}$ and hence we expect a larger value of $\xi_{s}$. We can then fairly assume that in all our samples $d \ll \xi_{s}$ such that fields are homogeneous in the $\mathrm{Al}$ adjacent to the EuS layer, as discussed in previous sections. The bottom Al-wire

\footnotetext{
${ }^{3}$ This unusual behavior of $T_{c}$ as a function of thickness is a known property of Al thin films [57].
}

TABLE I. Properties of the Al layer in contact with the EuS for the different fabricated samples. The averaged exchange field is extracted from the data at zero magnetic field. The last column gives the critical thickness obtained from Eq. (15). See main text for details.

\begin{tabular}{ccccc}
\hline \hline Sample & $d(\mathrm{~nm})$ & $\Delta_{2,0}(\mu \mathrm{eV})$ & $\bar{h}(\mu \mathrm{eV})$ & $d_{c}(\mathrm{~nm})$ \\
\hline S1 & 10 & 245 & 78.5 & 4.5 \\
S2 & 8 & 255 & 118.5 & 5.3 \\
S3 & 9 & 245 & 146 & 7.6 \\
S4 & 3 & 0 & No SC & 5.4 \\
\hline \hline
\end{tabular}

thickness was different for the four analyzed samples, see Table I. The top Al wires were 10-12 nm thick, depending on the sample.

Samples S1, S2, S3 show the characteristic large spin splitting of the Al layer in contact with the EuS, even at zero applied magnetic field. The typical $d I / d V$ obtained from I-V characteristics are shown in Fig. 5(b) (we show only data for sample S3). Sample S4 does not show a spin-split $d I / d V$ because the bottom Al layer is not superconducting. As we explain next, this is due to its small thickness.

By fitting the $d I / d V$ curves we can extract the values of the two Al gaps, $\Delta_{1,2}$ (top and bottom Al layers, respectively), the effective exchange field, $\bar{h}$ [11] (see Table I) and the spin-flip rate $1 / \bar{\tau}_{\text {sf }}$. The gap of the top Al layer, $\Delta_{1}$, can be determined from the $d I / d V$ at large magnetic fields when $\Delta_{2}$ is suppressed [see green curve in Fig. 5(b)]. For example for $\mathrm{S} 3$ we obtain $\Delta_{1,0} \approx 235 \mu \mathrm{eV}$. We determine from similar fitting the values of $\Delta_{2}$ and $\bar{h}$ at zero field: $\Delta_{2,0} \approx 245 \mu \mathrm{eV}, \bar{h} \approx 146 \mu \mathrm{eV}$. Here, $\Delta_{1,0}$ and $\Delta_{2,0}$ stand for the field-free order parameter. The real value of the order parameter is a bit smaller due to the exchange interaction. For example, at $B=0$, a self-consistent calculation gives a value of $\Delta_{2}=219 \mu \mathrm{eV}$, as shown in Fig. 5(c). In order to get a good agreement between the experimental and theoretical $d I / d V$, the effect of the magnetic impurities on the interface between EuS and the bottom Al layer is taken into account, with $\tau_{\text {sf }}^{-1} \approx 38.5 \mu \mathrm{eV}$. This corresponds to a spin relaxation time $\tau_{\mathrm{sf}} \sim 10 \mathrm{ps}$, much smaller than pure Al films. Provided that raw aluminum pellets used to grow the films are of 99.99 grade, the intrinsic level of impurities is below $0.01 \%$. Another source of magnetic impurities could be a diffusion of $\mathrm{Eu}$ on the interface. A recent paper unveiled that epitaxial films of aluminum grow without intermixing on the single crystal EuS, demonstrating atomically sharp interfaces [44]. Our samples feature polycrystalline $\mathrm{Al}$ and EuS films, therefore it was difficult to demonstrate experimentally that the interfaces are equally sharp. Nevertheless, our X-ray photoelectron spectroscopy data (not shown) reveal rapid attenuation of the $\mathrm{Eu}$ peaks with increasing of thickness of the $\mathrm{Al}$ overlayer, thus corroborating negligible level of magnetic impurities in the $\mathrm{Al}$ and proving that the main source of spin flipping is the interface. Because of the tunneling barrier, the top $\mathrm{Al}$ is not affected by the exchange field and therefore it has the usual BCS DoS.

All experimental data shown in Figs. 5(a)-5(b) were obtained in the low-temperature regime, $T=30 \mathrm{mK}$ such that 
(a)
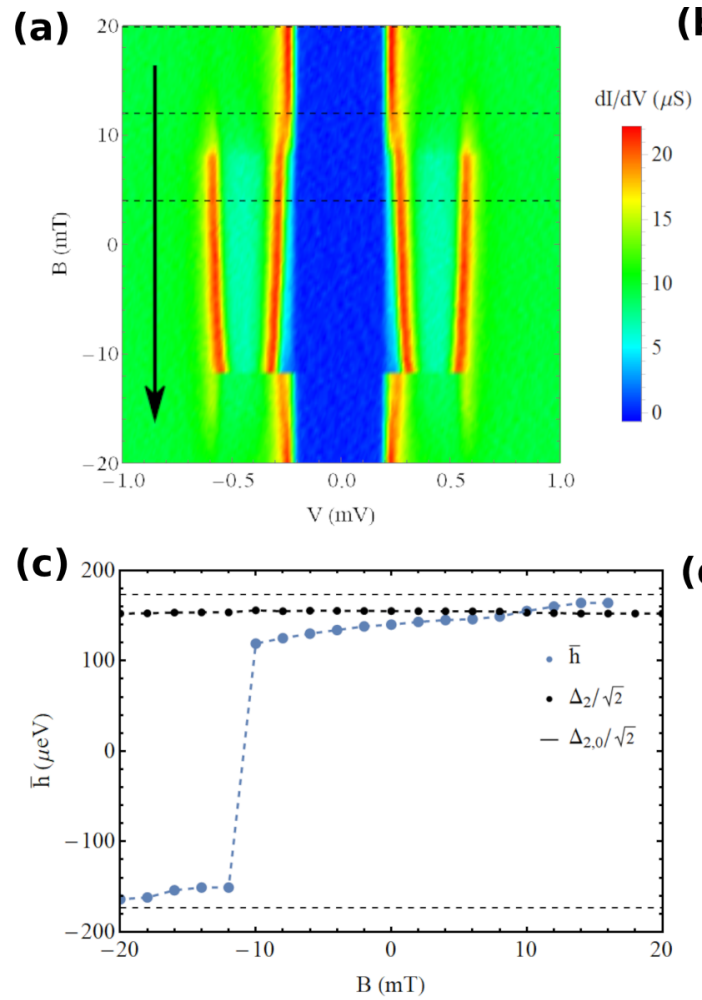

(b)

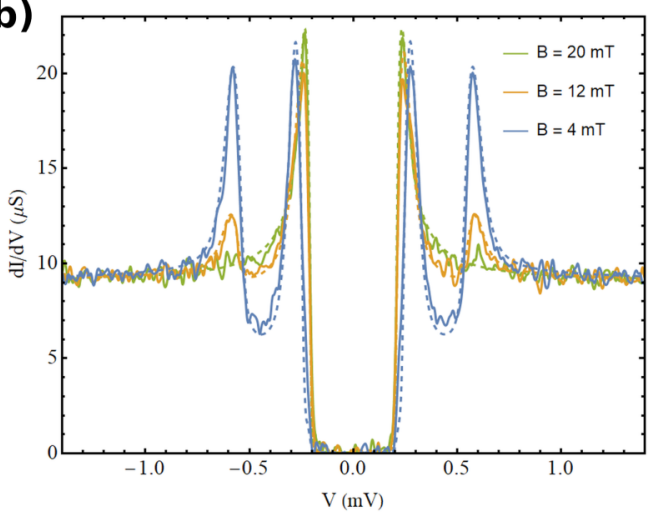

(d)

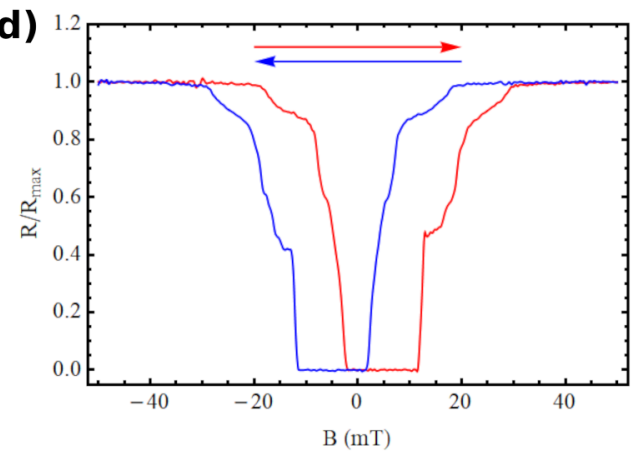

FIG. 5. (a) The differential conductance of the $\mathrm{S} 3$ sample measured at $30 \mathrm{mK}$ as a function of the external magnetic field and the voltage drop across the junction. The arrow indicates the direction of the magnetic field sweep. At $B=10 \mathrm{mT}$ most of the bottom Al film undergoes a transition into the superconducting state. (b) $d I / d V$ curves at three different values of $B(B=4, B=12$ and $B=20 \mathrm{mT})$, indicated by dashed lines in (a). The solid lines correspond to the experimental data, whereas the dashed lines to the theoretical fitting. The values of the fitting parameters used in the theoretical model at $B=0$ are $\bar{h}=146 \mu \mathrm{eV}, \Delta_{2}=219 \mu \mathrm{eV}$, and $\tau_{\mathrm{sf}}^{-1}=38.5 \mu \mathrm{eV}$. (c) Effective exchange field, $\bar{h}$, and self-consistent order parameter, $\Delta_{2}$ of the bottom Al layer divided by $\sqrt{2}$. The horizontal dashed lines represent the Chandrasekhar-Clogston critical field $\Delta_{2,0} / \sqrt{2}$ in the absence of magnetic impurities. (d) Magnetoresistance of the bottom Al film adjacent to the EuS layer measured at $T=30 \mathrm{mK}$.

$T \ll \Delta_{2}$. In this regime, according to the discussion in Sec. IV, one expects a first-order phase transition when $\bar{h} \approx$ $\Delta_{0} / \sqrt{2}$ when the spin-flip rate $1 / \tau_{\text {sf }}$ is sufficiently small. Using as a guide the $\bar{h}$ values obtained from the zero-magnetic field fitting and taking into account the averaged thickness of the bottom Al layer we calculate the critical thickness below which superconductivity should vanish from

$$
d_{c}=\sqrt{2} d \bar{h} / \Delta_{2,0} .
$$

The last column in Table I shows $d_{c}$ for all four samples. For the $\mathrm{S} 1-\mathrm{S} 3$ samples $d_{c}$ is close to, but smaller than the nominal thicknesses $d$ and the coexistence between superconductivity and spin splitting is allowed.

As we mentioned before, the sample S4 does not show the spin-split $d I / d V$ curve, and only the superconducting behavior of the top Al layer is detected. Since the four samples were prepared one after the other, we can assume that the EuS layer for this sample is similar to the other samples, and then we can fairly assume a similar interfacial exchange and, therefore we can calculate a critical thickness of $d_{c}=5.4 \mathrm{~nm}$. This value exceeds the nominal Al thickness grown experimentally, and explains why superconducting transition may not take place. In other words, these results confirm that when the $\mathrm{Al}$ thickness is smaller than $d_{c}$, a superconducting transition does not take place as discussed in previous sections.

Further conclusions can be drawn from the dependence of the $d I / d V$ curves on an external magnetic field $B$. Such field affects superconductivity in two ways: via Zeeman and orbital effects. Because all films in our samples are very thin, and the magnetic field is applied in plane, we can neglect the orbital effects [45] and focus on the dominant Zeeman interaction. The saturation magnetization of the EuS and parameters of the magnetization reversal are determined by the fabrication process of the layers [see the magnetization curve of Fig. 6(a)]. On the other hand, the interfacial exchange field that depends on the magnetic moment is controlled by external magnetic field. In Figs. 5(a)-5(b) we show the full evolution of the measured $d I / d V$ curves of sample $\mathrm{S} 3$ when the field is varied continuously from $+20 \mathrm{mT}$ to $-20 \mathrm{mT}$. At high positive magnetic fields, the EuS is homogeneously magnetized, the exchange field is maximized, and superconductivity cannot develop in the lower Al layer. Thus, the $d I / d V$ curve is basically proportional to the DoS of the upper Al layer, see green curve in Fig. 5(b), and the measured gap corresponds to the top Al layer $\left(\Delta_{1}\right)$. From Fig. $5(\mathrm{a})$ one sees that at $B \approx 10 \mathrm{mT}$, a second coherence peak appears. This indicates that the $\mathrm{Al}$ layer in contact with the EuS goes through a phase transition into the superconducting state. When the field changes sign, 

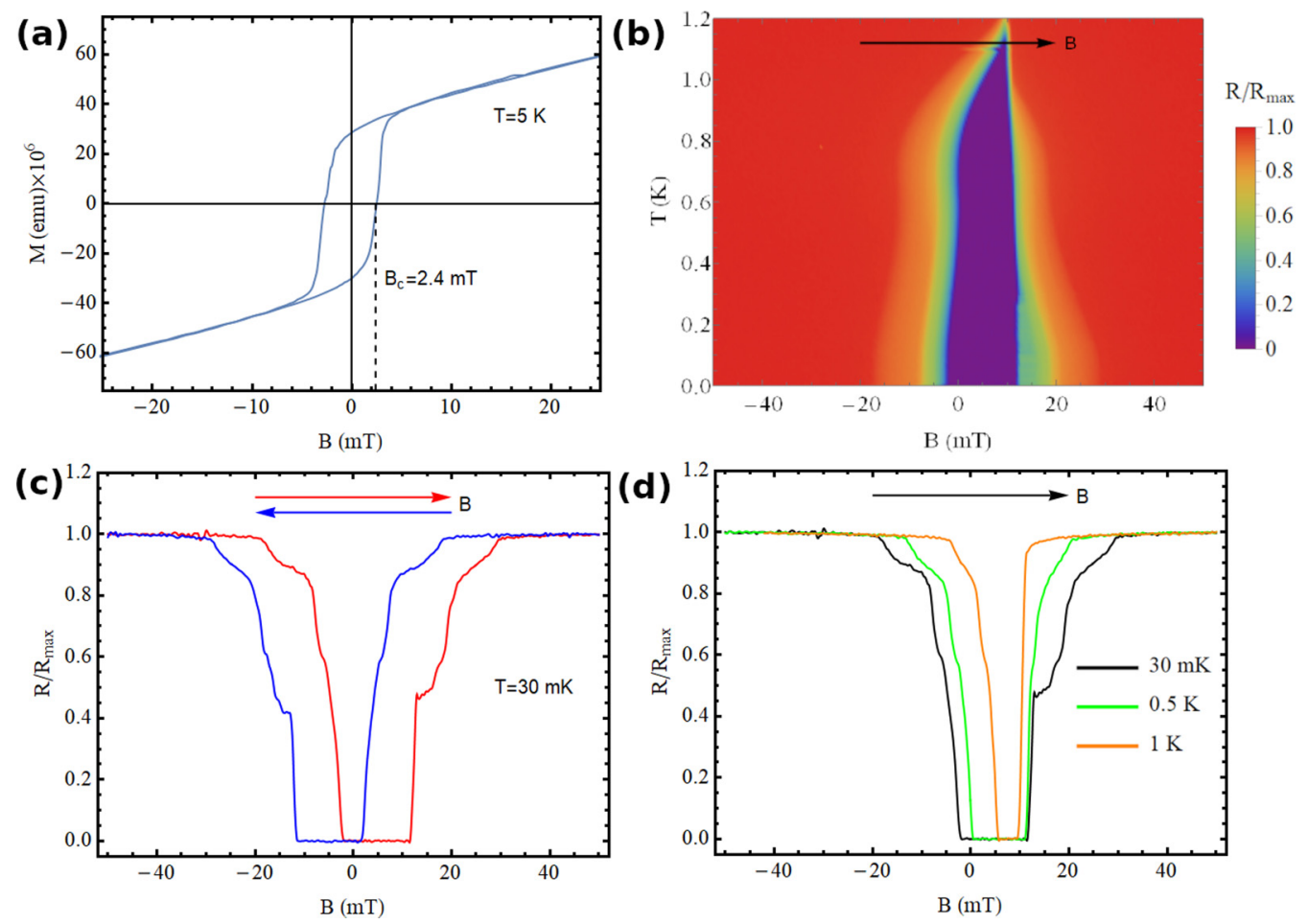

FIG. 6. (a) Magnetization loop measured for a continuous EuS film at $5 \mathrm{~K}$, with the field applied in the in-plane direction. (b) Contour plot showing the resistance of $\mathrm{Al}$ wire adjacent to the EuS layer as a function of the external field and the temperature. The arrow indicates the sweep direction of the magnetic field. These measurements and the measurements shown in the next two panels were performed on the sample S3. (c) The hysteresis of the resistance of the Al wire adjacent to the EuS layer at $T=30 \mathrm{mK}$. (d) The $B$-field dependence of the resistance of the $\mathrm{Al}$ wire adjacent to the EuS layer at different temperatures.

it is opposed to magnetization. Parts of the EuS film start to switch their magnetization weakening the average exchange field. This leads to the reduction of the spin splitting up to $B \approx$ $-12 \mathrm{mT}$. At this value we observe a sudden disappearance of the outer coherence peak. This value of the $B$ field corresponds to the switching of the EuS magnetization. The magnetic film is now almost homogeneously magnetized in the opposite direction and the resulting exchange field is strong enough to suppress superconductivity in the bottom layer. The $d I / d V$ reflects again the $\mathrm{DoS}$ of the $\mathrm{Al}$ at the top.

From our theoretical model we performed a fitting of several $d I / d V$ curves at different magnetic fields, see for example Fig. 5(b). From these fittings we extract the values of $\bar{h}$ and $\Delta_{2}$, as shown in Fig. 5(c). In the absence of magnetic disorder one expects the first-order phase transition at $\bar{h}_{c}=$ $\Delta_{2,0} / \sqrt{2}$. For S3 this would correspond to $\bar{h}_{c} \sim 173 \mu \mathrm{eV}$, shown in Fig. 5(c) with a dashed horizontal line. However, as mentioned before, the magnetic disorder in our samples is sizable and leads to a smaller value of $\Delta_{2}$ even at zero splitting field. This explains their smaller values, shown with black dots in Fig. 5(c). The values of $\bar{h}$ extracted from the fitting are shown with blue circles. $\bar{h}$ and $\Delta_{2} / \sqrt{2}$ cross at $B=10 \mathrm{mT}$, the field at which the main phase transition occurs [see Fig. 5(a)]. This suggests that the critical exchange field is indeed equal to $\Delta_{2,0}^{*} / \sqrt{2}$, where now $\Delta_{2,0}^{*}$ is the value of the gap at zero exchange field but in the presence of magnetic impurities.

There is an additional important feature in the results of Fig. 5. On the one hand, the gap is practically constant for all the field values below the critical exchange field, indicating a first-order phase transition. On the other hand, Fig. 5(a) exhibits traces of the outer peak at values of $B$ larger than the critical one $(B>10 \mathrm{mT})$. That peak disappears smoothly, as shown in Figs. 5(a)-5(b). This indicates that for fields $10<B<16 \mathrm{mT}$, the bottom Al layer is in a mixed phase, exhibiting superconducting and normal regions. This scenario is very likely given by small nonuniformity in the large cross section of the junction, $200 \mu \mathrm{m} \times 200 \mu \mathrm{m}$. We quantify the mixed phase regime by a parameter $u$, which is equal to 1 in the complete superconducting state and 0 in the normal state. The measured differential conductance is then the result of the average: $d I / d V=u d I /\left.d V\right|_{S S}+(1-u) d I /\left.d V\right|_{S N}$, where $S S(S N)$ denotes the conductance measured when the bottom $\mathrm{Al}$ layer is in the superconducting (normal) state. Using the parameter $u$ we have fitted the $d I / d V$ curve at $B=12 \mathrm{mT}$ and $B=20 \mathrm{mT}$, yellow and green curves in Fig. 5(b), by assuming $u=0.25$ and $u=0$, respectively. The agreement between theory and experiment is very good.

Two complementary scenarios can explain the mixed superconducting-normal state. A possible explanation for the smooth disappearance of the coherence peaks is that the bottom $\mathrm{Al}$ has a spatially nonconstant thickness. Fluctuations between $0.5-1 \mathrm{~nm}$, will result in parts of the samples that turn superconducting at field values at which other parts remain normal, cf. parameters for S3 in Table I. An additional possible scenario to explain the appearance of the mixed phase is the polycrystalline nature of the EuS films $[5,8]$. Indeed our EuS film may contain at the interface with $\mathrm{Al}$, 
grains of different sizes exhibiting superparamagnetism. At low temperatures, their magnetization direction is basically determined by the interaction with each other and the rest of the film via dipole and exchange interaction. As a consequence, one expects different values for the switching field, depending on their sizes and total magnetic moment. The distribution of grains with different magnetization directions causes a spatially inhomogeneous exchange field, which leads to a phase transition occurring at different values of the $\mathrm{B}$ field in different parts of the sample. The last scenario is also consistent with the smooth switching of the resistance of the Al layer as a function of the $B$ field, as shown in Fig. 5(d). The resistance changes in a steplike form, suggesting the presence of a few types of grains that switch their magnetization at different $B$-field values (a more detailed description of the magnetization reversal of the EuS film is presented in Appendix C).

It is also clear from our analysis that the strong $B$ dependence of the splitting field observed in our samples cannot be explained by the pure Zeeman field. A magnetic field of $20 \mathrm{mT}$ corresponds to Zeeman energy of $g \mu_{B} B / 2=1.16 \mu \mathrm{eV}$, where $g \approx 2$ is the Landé $g$ factor. This energy is more than an order of magnitude smaller than the (measured) decrease in the spin splitting that accompanies a decreasing of the field from 20-0 $\mathrm{mT}$ [see Fig. 5(c)]. Such a strong variation of the spin splitting is only due to the intrinsic properties of the EuS/Al interface. From a microscopic perspective, the interfacial exchange field $h$, and hence the splitting is determined by the spatial distribution of magnetic moments, and the value of the coupling between these moments and the spin of conduction band electrons in $\mathrm{Al}[25,46]$. We have studied how the growth conditions affect these parameters. Results of this investigation are consistent with earlier work [47] and will be published elsewhere. In general, we found that high vacuum conditions are crucial to protect the EuS layer from oxidization and to avoid a paramagnetic phase at the EuS/Al interface. On the other hand, the sublimation temperature of the EuS powder defines the stoichiometry of the resulting EuS films. Nonoptimal conditions were yielding samples with excess of Eu that strongly increases both the interfacial exchange field and the spin-flip relaxation rate, and above a certain critical concentration quenches the superconductivity in the adjacent $\mathrm{Al}$ wire.

\section{CONCLUSION}

We have presented an exhaustive study of the role of the superconductor thickness on the spectral properties and the critical exchange field of a FI/S bilayer. We found that the exchange field produces a well-defined spin splitting for thin and intermediate sized superconductors. The splitting is, however, suppressed away from the FI/S interface in thick superconductors. Moreover, the spin splitting at the FI/S interface is well approximated by the effective exchange field (7) even for thick samples. We have also studied the effect of the spin-flip relaxation due to magnetic impurities localized at the interface on the spectral properties of the system. The spin-flip relaxation suppresses the spin splitting and lifts the superconducting gap, reducing the critical exchange field. We have also studied the nature of the superconducting phase transition as a function of the exchange field and the $\mathrm{S}$ layer thickness. We found that thicker samples favor second-order phase transitions. In other words, we show that the thickness of the superconducting layer determines the order of the phase transition. We have also found a temperature-dependent thickness above which the critical surface field diverges, and no phase transition occurs, regardless of the value of the exchange field.

One of the important conclusions is that the coexistence between superconductivity and spin-splitting fields in thin layers is subject to a subtle balance between the thickness of the film and the interfacial exchange field determined by the quality of the FI/S interface and its magnetic properties. Variations of the order of a few $\mathrm{nm}$ in the thickness of the $\mathrm{S}$ film may prevent the appearance of superconductivity.

We have contrasted our theoretical findings with experiments on EuS/Al samples fabricated by us. Our samples show a well-defined spin-split density of states. One of the samples with the thinnest Al film shows no superconductivity at $30 \mathrm{mK}$, in agreement with our theoretical prediction for thin films. In the other samples with a robust superconducting state, we found clear evidence of a first-order phase transition. We also found evidence of a coexistence phase regime where some part of the sample is superconducting, while the rest stays in the normal state. This mixed phase is attributed to the spatial fluctuation of the $\mathrm{Al}$ thickness, and a grainy texture of the magnetic EuS/Al interface.

Our results provide important insights for the fabrication of $\mathrm{FI} / \mathrm{S}$ structures essential for applications where the control of a spin splitting in a superconductor is crucial $[9,16,24,48-52]$. Our analysis shows that both the thickness of the S layer and the interfacial exchange field, given by the interface quality, are important quantities that have to be carefully chosen in the fabrication process.

Note added. Recently, there was another related paper, Ref. [53], in which the authors studied a thin superconductor/ferromagnetic insulator bilayer, and explored the engineering of topological superconductivity in an adjacent semiconducting nanowire. To compute the density of states and order parameter they employed a very similar approach based on the Usadel equation as the one in the present paper.

\section{ACKNOWLEDGMENTS}

We thank Jagadeesh Moodera for useful discussions. F.S.B. acknowledges funding by the Spanish Ministerio de Ciencia, Innovación y Universidades (MICINN) (Project FIS201782804-P). T.T.H. acknowledges funding from the Academy of Finland (Project number 317118). A.H. acknowledges funding by the Department of Education of the Basque Government (Ikasiker grant). C.G.-O. acknowledges funding of the Ph.D. fellowship from MPC foundation. S.K. acknowledges for the fellowship of the ICTP Program for Training and Research in Italian laboratories, Trieste, Italy. C.R. acknowledges support from Gobierno Vasco (Grant No. IT 1255-19). F.G. acknowledges the European Research Council under the EU's Horizon 2020 Grant Agreement No. 899315-TERASEC for partial financial support. We acknowledge funding from EU's Horizon 2020 research and innovation program under 
Grant Agreement No. 800923 (SUPERTED). F.S.B. thanks the Institute of Solid State Theory at University of Münster for its kind hospitality.

\section{APPENDIX A: MULTIMODE METHOD ANSATZ}

To solve the linearized Usadel equation (10) we use the multimode method [41]. $\hat{f}$ is diagonal in spin space because $\boldsymbol{h}$ points in a single direction, so it can be written as

$$
\hat{f}=i f_{0}+f_{3} \sigma_{3} .
$$

Here $f_{0}$ and $f_{3}$ describe singlet and triplet pairs, respectively.

From Eq. (10), we obtain that the Usadel equations for $f_{0}$ and $f_{3}$ are

$$
\begin{gathered}
\xi_{s}^{2} \pi T_{c 0} \partial_{x x}^{2} f_{0}-\left|\omega_{n}\right| f_{0}+\Delta=0, \\
\xi_{s}^{2} \pi T_{c 0} \partial_{x x}^{2} f_{3}-\left|\omega_{n}\right| f_{3}=0 .
\end{gathered}
$$

The boundary conditions are

$$
\begin{gathered}
\left.\partial_{x} f_{0}\right|_{x=0}=\operatorname{sgn}\left(w_{n}\right) \kappa_{h} f_{3}+\left.6 \kappa_{\text {sf }} f_{0}\right|_{x=0}, \\
\left.\partial_{x} f_{3}\right|_{x=0}=-\operatorname{sgn}\left(w_{n}\right) \kappa_{h} f_{0}+\left.2 \kappa_{\text {sf }} f_{3}\right|_{x=0}, \\
\left.\partial_{x} f_{0}\right|_{x=d}=0, \\
\left.\partial_{x} f_{3}\right|_{x=d}=0 .
\end{gathered}
$$

Since $\Delta$ is spin independent, the singlet component is an even function of $\omega_{n}, f_{0}\left(-\omega_{n}\right)=f_{0}\left(\omega_{n}\right)$, and the triplet component is an odd function of $\omega_{n}, f_{3}\left(-\omega_{n}\right)=-f_{3}\left(\omega_{n}\right)[54,55]$. Therefore, the self-consistency equation (12) can be rewritten in terms of the singlet component

$$
\Delta \ln \frac{T_{c 0}}{T}=2 \pi T \sum_{\omega_{n}>0}\left(\frac{\Delta}{\omega_{n}}-f_{0}\right) .
$$

In the multimode approach, one seeks as solution to Eqs. (A2)-(A8) in the form [41]

$$
\begin{array}{r}
f_{0}\left(x, \omega_{n}\right)=F_{0}\left(\omega_{n}\right) \cos \left(\Omega_{0} \frac{x-d}{\xi_{s}}\right) \\
+\sum_{m=1}^{\infty} F_{m}\left(\omega_{n}\right) \frac{\cosh \left(\Omega_{m} \frac{x-d}{\xi_{s}}\right)}{\cosh \left(\Omega_{m} \frac{d}{\xi_{s}}\right)} \\
f_{3}\left(x, \omega_{n}\right)=F\left(\omega_{n}\right) \cosh \left(\Omega_{z} \frac{x-d}{\xi_{s}}\right) \\
\Delta(x)=\delta_{0} \cos \left(\Omega_{0} \frac{x-d}{\xi_{s}}\right)+\sum_{m=1}^{\infty} \delta_{m} \frac{\cosh \left(\Omega_{m} \frac{x-d}{\xi_{s}}\right)}{\cosh \left(\Omega_{m} \frac{d}{\xi_{s}}\right)},
\end{array}
$$

where $\Omega_{z}^{2}=\omega_{n} /\left(\pi T_{c 0}\right)$. This ansatz automatically satisfies the boundary conditions at $x=d$.

The relation between the variables $\delta_{m}$ and $F_{m}$ is derived from (A2). Solving $F$ from the B.C. equations at $x=0$, we obtain a set of linear homogeneous equations for the order parameter amplitudes $\delta_{m}$ :

$$
K_{n m} \delta_{m}=0,
$$

where

$$
\begin{aligned}
K_{n 0}= & \left(\frac{\Omega_{0}}{\xi_{s}} \sin \frac{\Omega_{0} d}{\xi_{s}}-6 \kappa_{\mathrm{sf}} \cos \frac{\Omega_{0} d}{\xi_{s}}\right. \\
& \left.-\frac{\kappa_{h}^{2} \cos \frac{\Omega_{0} d}{\xi_{s}} \cosh \frac{\Omega_{z} d}{\xi_{s}}}{\frac{\Omega_{z}}{\xi_{s}} \sinh \frac{\Omega_{z} d}{\xi_{s}}+2 \kappa_{\mathrm{sf}} \cosh \frac{\Omega_{z} d}{\xi_{s}}}\right) /\left(\omega_{n}+\Omega_{0}^{2} \pi T_{c 0}\right),
\end{aligned}
$$

$$
\begin{aligned}
K_{n m}= & \left(-\frac{\Omega_{m}}{\xi_{s}} \tanh \frac{\Omega_{m} d}{\xi_{s}}-6 \kappa_{\mathrm{sf}}\right. \\
& \left.-\frac{\kappa_{h}^{2} \cosh \frac{\Omega_{z} d}{\xi_{s}}}{\frac{\Omega_{z}}{\xi_{s}} \sinh \frac{\Omega_{z} d}{\xi_{s}}+2 \kappa_{\mathrm{sf}} \cosh \frac{\Omega_{z} d}{\xi_{s}}}\right) /\left(\omega_{n}-\Omega_{m}^{2} \pi T_{c 0}\right),
\end{aligned}
$$

$m \geqslant 1$.

The mode frequencies $\Omega_{m}$ are determined by the selfconsistency equation (A8).

Numerically, one can obtain $\kappa_{h}$ from Eq. (A12) by considering the same amount of Matsubara frequencies and modes. The critical exchange field is then obtained by solving the equation

$$
\operatorname{det} \hat{K}=0 .
$$

The system of Eq. (A14) might have multiple solutions for the critical temperature. For a given exchange field and thickness of the superconductor, the critical temperature is determined as the largest solution [41].

\section{APPENDIX B: DERIVATION OF THE THICKNESS $\boldsymbol{d}^{*}$}

In this Appendix we study the critical exchange field in the absence of magnetic impurities at $T=0$ and obtain an analytical expression for the thickness $d^{*}$ at which it diverges. The critical exchange field is obtained using the multimode method introduced in Sec. IV.

Taking the asymptotic expansion of the self-consistency equation (A8) in the $T \rightarrow 0$ limit, we obtain that the mode frequencies $\Omega_{m}$ are

$$
\begin{gathered}
\Omega_{0}^{2} \sim \frac{1}{2 \gamma_{E}} \\
\Omega_{m}^{2} \sim \frac{T}{T_{c 0}}(2 m-1), \quad m \geqslant 1,
\end{gathered}
$$

where $\gamma_{E} \approx 1.781$ is the exponent of the Euler-Mascheroni constant.

Substituting this value in Eq. (A13), we obtain that the elements of $\hat{K}$ to lowest order in $T / T_{c 0}$ are

$$
\begin{aligned}
& K_{n 0} \propto 1-\frac{(2 n+1) T}{T_{c 0}}\left(1+\frac{\Omega_{0} d}{\kappa_{h}^{2} \xi_{s}^{3}} \tan \frac{\Omega_{0} d}{\xi_{s}}\right) \\
& K_{n m} \propto \frac{1}{(n-m+1)}\left(1+\frac{(2 n+1)(2 m-1) d^{2}}{\kappa_{h}^{2} \xi_{s}^{4}}\left(\frac{T}{T_{c 0}}\right)^{2}\right) .
\end{aligned}
$$

The term $\tan \left(\Omega_{0} d / \xi_{s}\right)$ in Eq. (B3a) diverges when $\Omega_{0} d / \xi_{s}=\pi / 2$. It can be proven that the value of $\Omega_{0}$ lies in the interval $0<\Omega_{0}^{2}<1 /\left(2 \gamma_{E}\right)$, so the values of $d$ for which 
determinant (A14) diverges are

$$
\sqrt{\frac{1}{2 \gamma_{E}}} \frac{d}{\xi_{s}} \geqslant \frac{\pi}{2} .
$$

Therefore, the maximum thickness for which the critical exchange field exists is

$$
d^{*}=\sqrt{\frac{\gamma_{E}}{2}} \pi \xi_{s} \approx 3.0 \xi_{s} .
$$

For thicknesses greater than $d^{*}$, the critical exchange field will diverge at some finite temperature, so at $T=0$ the system will be in the superconducting state regardless of the magnitude of the exchange field.

\section{APPENDIX C: MAGNETIC PROPERTIES OF THE EuS FILMS}

Magnetic properties of the thin films of EuS were studied in several works. To check the consistency of our data, we have grown a $12 \mathrm{~nm}$ thick film of EuS on the polished fused silica substrate under the same conditions used in the fabrication of the tunnel junctions. Figure 6(a) shows the magnetization loop measured at $5 \mathrm{~K}$ in the magnetic field applied parallel to the film. Magnetization reversal occurs at the coercive field, $2.4 \mathrm{mT}$. The film has a large value of the remanent magnetization similar to what was observed for the samples of Moodera's group [5]. Nevertheless, magnetization grows slowly and saturates only in the field of a few Tesla. It implies that a polycrystalline EuS includes a certain amount of superparamagnetic grains weakly coupled to the main film. The relatively small value of the coercive field corroborates this observation. Indeed, for the magnetization reversal dominated by the domain walls movement, the coercive field increases with the size of the magnetic irregularities. Therefore, fine polycrystalline structure results in lower coercive fields [5]. Comparing the magnetization loop presented in Fig. 6(a) with the data reported in Ref. [5], it is clear that the coercive field in our sample is similar to the coercive field of thin EuS films grown at $77 \mathrm{~K}$, which were found to have grains with $4.4 \mathrm{~nm}$ diameters. Since EuS has no magnetocrystalline anisotropy, it is reasonable to expect very low values of the blocking temperature for the small superparamagnetic grains [56]. We could not measure magnetization loops below $1 \mathrm{~K}$. However, the dependence of the bottom Al layer's resistance interfaced with EuS film on the magnetic field provide indirect confirmation of this conjecture. At $30 \mathrm{mK}$, all superparamagnetic particles are in the blocked state. The $R(B)$ curve shows distinctive jumps that can be interpreted as magnetization reversal of the components with different magnetic anisotropy [Fig. 6(c)], as we explain next. If the exchange coupling between the grains is weak, the orientation of their magnetic moments depends on the dipolar interaction with the rest of the film and with the coupling with the external field. Whereas the external field will try to align the particles' moment, the dipolar interaction tries to orient the moment of the particles in the direction opposite to the magnetic moment of the film. Considering a descending branch of the resistance [red curve in Fig. 6(c)], we can see that all moments are aligned at a high positive field, and the effective exchange field is high (no superconductivity). When the field decreases down to zero, the dipolar interaction dominates. The resistance decreases in steps that correspond to consecutive switching of the magnetization of the superparamagnetic particles. The average magnetic moment becomes smaller and leads to a decrease in the effective exchange field. This decrease allows the Al wire to become superconducting. The zero-resistance state remains up to small negative values of the field. Further increase of the negative magnetic field leads again to consecutive reversal of the particles' magnetic moments accompanied by the switching of the total magnetic moment. The effective exchange field is large again and quenches superconductivity. This gives rise to the increase of the resistance. The same measurements performed at a higher temperature [Fig. 6(d)] show that the critical fields corresponding to the reversal of the superparamagnetic particles progressively disappear, showing the transition to the unblocked state.
[1] J. S. Moodera, X. Hao, G. A. Gibson, and R. Meservey, Electron-Spin Polarization in Tunnel Junctions in Zero Applied Field with Ferromagnetic EuS Barriers, Phys. Rev. Lett. 61, 637 (1988).

[2] X. Hao, J. S. Moodera, and R. Meservey, Spin-filter effect of ferromagnetic europium sulfide tunnel barriers, Phys. Rev. B 42, 8235 (1990).

[3] X. Hao, J. S. Moodera, and R. Meservey, Thin-Film Superconductor in an Exchange Field, Phys. Rev. Lett. 67, 1342 (1991).

[4] J. S. Moodera, T. S. Santos, and T. Nagahama, The phenomena of spin-filter tunnelling, J. Phys.: Condens. Matter 19, 165202 (2007).

[5] G.-X. Miao and J. S. Moodera, Controlling magnetic switching properties of EuS for constructing double spin filter magnetic tunnel junctions, Appl. Phys. Lett. 94, 182504 (2009).

[6] Y. M. Xiong, S. Stadler, P. W. Adams, and G. Catelani, SpinResolved Tunneling Studies of the Exchange Field in EuS/Al Bilayers, Phys. Rev. Lett. 106, 247001 (2011).
[7] B. Li, G.-X. Miao, and J. S. Moodera, Observation of tunnel magnetoresistance in a superconducting junction with Zeeman-split energy bands, Phys. Rev. B 88, 161105(R) (2013).

[8] E. Strambini, V. N. Golovach, G. De Simoni, J. S. Moodera, F. S. Bergeret, and F. Giazotto, Revealing the magnetic proximity effect in EuS/Al bilayers through superconducting tunneling spectroscopy, Phys. Rev. Mater. 1, 054402 (2017).

[9] G. De Simoni, E. Strambini, J. S. Moodera, F. S. Bergeret, and F. Giazotto, Toward the absolute spin-valve effect in superconducting tunnel junctions, Nano Lett. 18, 6369 (2018).

[10] M. Rouco, S. Chakraborty, F. Aikebaier, V. N. Golovach, E. Strambini, J. S. Moodera, F. Giazotto, T. T. Heikkilä, and F. S. Bergeret, Charge transport through spin-polarized tunnel junction between two spin-split superconductors, Phys. Rev. B 100, 184501 (2019).

[11] R. Meservey and P. Tedrow, Spin-polarized electron tunneling, Phys. Rep. 238, 173 (1994). 
[12] Y. Oreg, G. Refael, and F. von Oppen, Helical Liquids and Majorana Bound States in Quantum Wires, Phys. Rev. Lett. 105, 177002 (2010).

[13] R. M. Lutchyn, J. D. Sau, and S. Das Sarma, Majorana Fermions and a Topological Phase Transition in Semiconductor-Superconductor Heterostructures, Phys. Rev. Lett. 105, 077001 (2010).

[14] G.-X. Miao, J. Chang, B. A. Assaf, D. Heiman, and J. S. Moodera, Spin regulation in composite spin-filter barrier devices, Nat. Commun. 5, 3682 (2014).

[15] F. Giazotto, T. T. Heikkilä, A. Luukanen, A. M. Savin, and J. P. Pekola, Opportunities for mesoscopics in thermometry and refrigeration: Physics and applications, Rev. Mod. Phys. 78, 217 (2006).

[16] F. Giazotto, P. Solinas, A. Braggio, and F. S. Bergeret, Ferromagnetic-Insulator-Based Superconducting Junctions as Sensitive Electron Thermometers, Phys. Rev. Appl. 4, 044016 (2015).

[17] M. Alidoust, K. Halterman, and J. Linder, Singlet-triplet superconducting quantum magnetometer, Phys. Rev. B 88, 075435 (2013).

[18] E. Strambini, F. Bergeret, and F. Giazotto, Mesoscopic Josephson junctions with switchable current-phase relation, Europhys. Lett. 112, 17013 (2015).

[19] F. Giazotto and F. Bergeret, Very large thermal rectification in ferromagnetic insulator-based superconducting tunnel junctions, Appl. Phys. Lett. 116, 192601 (2020).

[20] F. Giazotto, T. T. Heikkilä, and F. S. Bergeret, Very Large Thermophase in Ferromagnetic Josephson Junctions, Phys. Rev. Lett. 114, 067001 (2015).

[21] F. Giazotto and F. Bergeret, Phase-tunable colossal magnetothermal resistance in ferromagnetic Josephson valves, Appl. Phys. Lett. 102, 132603 (2013).

[22] P. Machon, M. Eschrig, and W. Belzig, Nonlocal Thermoelectric Effects and Nonlocal Onsager Relations in a ThreeTerminal Proximity-Coupled Superconductor-Ferromagnet Device, Phys. Rev. Lett. 110, 047002 (2013).

[23] A. Ozaeta, P. Virtanen, F. S. Bergeret, and T. T. Heikkilä, Predicted Very Large Thermoelectric Effect in FerromagnetSuperconductor Junctions in the Presence of a Spin-Splitting Magnetic Field, Phys. Rev. Lett. 112, 057001 (2014).

[24] T. T. Heikkilä, R. Ojajärvi, I. J. Maasilta, E. Strambini, F. Giazotto, and F. S. Bergeret, Thermoelectric Radiation Detector Based on Superconductor-Ferromagnet Systems, Phys. Rev. Appl. 10, 034053 (2018).

[25] X.-P. Zhang, F. S. Bergeret, and V. N. Golovach, Theory of spin hall magnetoresistance from a microscopic perspective, Nano Lett. 19, 6330 (2019).

[26] T. Tokuyasu, J. A. Sauls, and D. Rainer, Proximity effect of a ferromagnetic insulator in contact with a superconductor, Phys. Rev. B 38, 8823 (1988).

[27] B. S. Chandrasekhar, A note on the maximum critical field of high-field superconductors, Appl. Phys. Lett. 1, 7 (1962).

[28] A. M. Clogston, Upper Limit for the Critical field in Hard Superconductors, Phys. Rev. Lett. 9, 266 (1962).

[29] G. Sarma, On the influence of a uniform exchange field acting on the spins of the conduction electrons in a superconductor, J. Phys. Chem. Solids 24, 1029 (1963).

[30] K. Maki and T. Tsuneto, Pauli Paramagnetism and Superconducting State, Prog. Theor. Phys. 31, 945 (1964).
[31] G. Eilenberger, Transformation of Gorkov's equation for type II superconductors into transport-like equations, Z. Phys. 214, 195 (1968).

[32] A. Larkin and Y. N. Ovchinnikov, Quasiclassical method in the theory of superconductivity, Zh. Eksp. Teor. Fiz. 55, 2262 (1969).

[33] K. D. Usadel, Generalized Diffusion Equation for Superconducting Alloys, Phys. Rev. Lett. 25, 507 (1970).

[34] A. Volkov, A. Zaitsev, and T. Klapwijk, Proximity effect under nonequilibrium conditions in double-barrier superconducting junctions, Physica C 210, 21 (1993).

[35] C. Lambert and R. Raimondi, Phase-coherent transport in hybrid superconducting nanostructures, J. Phys.: Condens. Matter 10, 901 (1998).

[36] W. Belzig, F. K. Wilhelm, C. Bruder, G. Schön, and A. D. Zaikin, Quasiclassical Green's function approach to mesoscopic superconductivity, Superlattices Microstruct. 25, 1251 (1999).

[37] F. S. Bergeret, M. Silaev, P. Virtanen, and T. T. Heikkilä, Colloquium: Nonequilibrium effects in superconductors with a spin-splitting field, Rev. Mod. Phys. 90, 041001 (2018).

[38] T. T. Heikkilä, M. Silaev, P. Virtanen, and F. S. Bergeret, Thermal, electric and spin transport in superconductor/ferromagnetic-insulator structures, Prog. Surf. Sci. 94, 100540 (2019).

[39] A. A. Abrikosov, L. Gorkov, and I. E. Dzyaloshinski, Methods of Quantum Field Theory in Statistical Physics, Second Edition (Pergamon Press, Oxford, 1965), Chap. 3.

[40] N. Kopnin, Theory of Nonequilibrium Superconductivity (Oxford Science Publications, Oxford, 2001).

[41] Y. V. Fominov, N. M. Chtchelkatchev, and A. A. Golubov, Nonmonotonic critical temperature in superconductor/ferromagnet bilayers, Phys. Rev. B 66, 014507 (2002).

[42] P. Virtanen, A. Vargunin, and M. Silaev, Quasiclassical free energy of superconductors: Disorder-driven first-order phase transition in superconductor/ferromagnetic-insulator bilayers, Phys. Rev. B 101, 094507 (2020).

[43] G.-X. Miao, K. S. Yoon, T. S. Santos, and J. S. Moodera, Influence of Spin-Polarized Current on Superconductivity and the Realization of Large Magnetoresistance, Phys. Rev. Lett. 98, 267001 (2007).

[44] Y. Liu, S. Vaitiekenas, S. Martí-Sánchez, C. Koch, S. Hart, Z. Cui, T. Kanne, S. A. Khan, R. Tanta, S. Upadhyay, M. E. Cachaza, C. M. Marcus, J. Arbiol, K. A. Moler, and P. Krogstrup, Semiconductor-ferromagnetic insulatorsuperconductor nanowires: Stray field and exchange field, Nano Lett. 20, 456 (2020).

[45] P. Fulde, High field superconductivity in thin films, Adv. Phys. 22, 667 (1973).

[46] J. M. Gomez-Perez, X.-P. Zhang, F. Calavalle, M. Ilyn, C. González-Orellana, M. Gobbi, C. Rogero, A. Chuvilin, V. N. Golovach, L. E. Hueso, F. S. Bergeret, and F. Casanova, Strong interfacial exchange field in a heavy metal/ferromagnetic insulator system determined by spin hall magnetoresistance, Nano Lett. 20, 6815 (2020).

[47] C. J. P. Smits, A. T. Filip, J. T. Kohlhepp, H. J. M. Swagten, B. Koopmans, and W. J. M. de Jonge, Magnetic and structural properties of EuS for magnetic tunnel junction barriers, J. Appl. Phys. 95, 7405 (2004).

[48] J. Linder and J. W. Robinson, Superconducting spintronics, Nat. Phys. 11, 307 (2015). 
[49] M. Eschrig, Spin-polarized supercurrents for spintronics: A review of current progress, Rep. Prog. Phys. 78, 104501 (2015).

[50] P. Virtanen, F. S. Bergeret, E. Strambini, F. Giazotto, and A. Braggio, Majorana bound states in hybrid two-dimensional Josephson junctions with ferromagnetic insulators, Phys. Rev. B 98, 020501(R) (2018).

[51] S. Manna, P. Wei, Y. Xie, K. T. Law, P. A. Lee, and J. S. Moodera, Signature of a pair of Majorana zero modes in superconducting gold surface states, Proc. Natl. Acad. Sci. 117, 8775 (2020).

[52] S. Vaitiekėnas, Y. Liu, P. Krogstrup, and C. M. Marcus, Zerobias peaks at zero magnetic field in ferromagnetic hybrid nanowires, Nat. Phys. 17, 43 ( 2021).

[53] A. Khindanov, J. Alicea, P. Lee, W. S. Cole, and A. E. Antipov, Topological superconductivity in nanowires proximate to a diffusive superconductormagnetic-insulator bilayer, Phys. Rev. B 103, 134506 (2021)

[54] F. S. Bergeret, A. F. Volkov, and K. B. Efetov, Odd triplet superconductivity and related phenomena in superconductorferromagnet structures, Rev. Mod. Phys. 77, 1321 (2005).
[55] F. S. Bergeret, A. F. Volkov, and K. B. Efetov, Odd triplet superconductivity in superconductor-ferromagnet hybrid structures, C. R. Phys. 7, 128 (2006).

[56] R. O'Hendley, Modern Magnetic Materials: Principles and Applications (John Wiley \& Sons, Inc., New York, 2000).

[57] P. N. Chubov, V. V. Eremenko, and Y. A. Pilipenko, Dependence of the critical temperature and energy gap on the thickness of superconducting aluminum films, Sov. Phys. JETP 28, 389 (1969).

[58] L. Aslamazov, Influence of impurities on the existence of an inhomogeneous state in a ferromagnetic superconductor, Sov. Phys. JETP 28, 773 (1969).

[59] S. Mironov, A. Mel'nikov, and A. Buzdin, Vanishing Meissner effect as a Hallmark of in-Plane Fulde-Ferrell-LarkinOvchinnikov Instability in Superconductor-Ferromagnet Layered Systems, Phys. Rev. Lett. 109, 237002 (2012).

[60] S. V. Mironov, D. Y. Vodolazov, Y. Yerin, A. V. Samokhvalov, A. S. Mel'nikov, and A. Buzdin, Temperature Controlled FuldeFerrell-Larkin-Ovchinnikov Instability in SuperconductorFerromagnet Hybrids, Phys. Rev. Lett. 121, 077002 (2018). 